\section{Pacific Northwest}

National Laboratory

Operated by Battelle for the

U.S. Department of Energy

\title{
Progress Report on the Advanced Large-Area Plastic Scintillators (ALPS) Project
}

\author{
D. V. Jordan \\ B. D. Geelhood \\ P. L. Reeder \\ D. L. Stephens, Jr. \\ R. C. Craig \\ J. I. McIntyre
}

May 2003

Prepared for the U.S. Department of Energy under Contract DE-AC06-76RL01830 


\title{
DISCLAIMER
}

This report was prepared as an account of work sponsored by an agency of the United States Government. Neither the United States Government nor any agency thereof, nor Battelle Memorial Institute, nor any of their employees, makes any warranty, express or implied, or assumes any legal liability or responsibility for the accuracy, completeness, or usefulness of any information, apparatus, product, or process disclosed, or represents that its use would not infringe privately owned rights. Reference herein to any specific commercial product, process, or service by trade name, trademark, manufacturer, or otherwise does not necessarily constitute or imply its endorsement, recommendation, or favoring by the United States Government or any agency thereof, or Battelle Memorial Institute. The views and opinions of authors expressed herein do not necessarily state or reflect those of the United States Government or any agency thereof.

\author{
PACIFIC NORTHWEST NATIONAL LABORATORY \\ operated by \\ BATTELLE \\ for the \\ UNITED STATES DEPARTMENT OF ENERGY \\ under Contract DE-AC06-76RL01830
}

This document was printed on recycled paper. 
PNNL-14283

\title{
Progress Report on the Advanced Large-Area Plastic Scintillators (ALPS) Project
}

\author{
D. V. Jordan \\ B. D. Geelhood \\ P. L. Reeder \\ D. L. Stephens, Jr. \\ R. C. Craig \\ J. I. McIntyre
}

May 2003

Prepared for the U.S. Department of Energy

under Contract DE-AC06-76RL01830

Pacific Northwest National Laboratory

Richland, Washington 99352 
PNNL-14283 


\section{Summary}

The U.S. Department of Energy tasked Pacific Northwest National Laboratory to investigate possible technological avenues for substantially advancing the state-of-the-art in gamma detection via large-area plastic scintillators. This letter report describes progress to date on this project. Early phases of the project, which commenced in January 2003, have focused on (1) quantifying the light-collection efficiency in plastic scintillator sheets as a function of photomultiplier tube positioning and edge-area coverage, (2) developing a conceptual design for a demonstration sensor, and (3) conducting initial laboratory setup and preliminary experiments using relatively small plastic scintillators for readout technique development and benchmarking of the modeling studies. 



\section{Acronyms}

$\begin{array}{ll}\text { ADC } & \text { analog to digital converter } \\ \text { ALPS } & \text { advanced large-area plastic scintillators } \\ \text { CAMAC } & \text { Computer Automated Measurement and Control } \\ \text { HPGe } & \text { high-purity germanium } \\ \text { LAI } & \text { limited-area island } \\ \text { LED } & \text { light-emitting diode } \\ \text { NIM } & \text { Nuclear Instrument Module } \\ \text { PET } & \text { positron emission tomography } \\ \text { PMT } & \text { photomultiplier tube } \\ \text { PNNL } & \text { Pacific Northwest National Laboratory } \\ \text { QDC } & \text { charge-integrating ADC } \\ \text { TDC } & \text { time-to-digital converter }\end{array}$





\section{Contents}

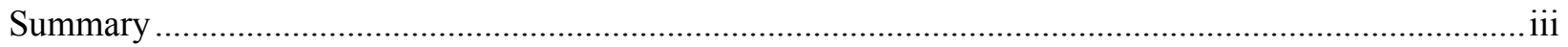

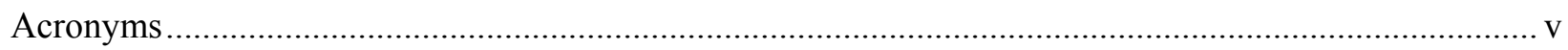

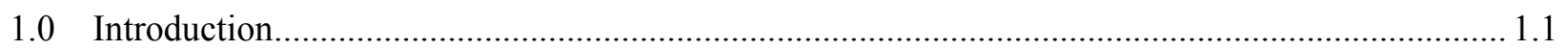

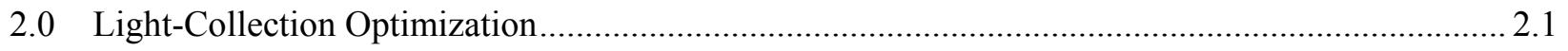

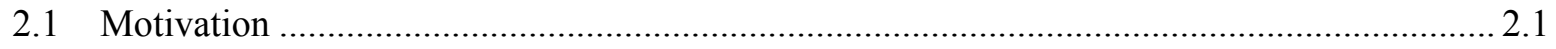

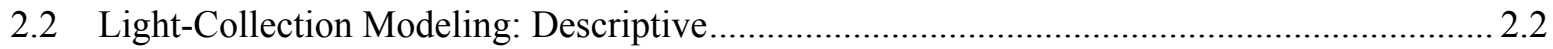

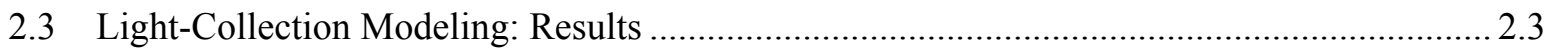

2.4 Light-Collection Modeling: Summary ....................................................................... 2.7

3.0 ALPS Conceptual Design and Experimental Campaign............................................................ 3.1

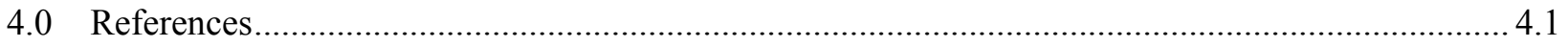

5.0 Appendix: Light-Collection Efficiency Scaling Effect............................................................. 5.1

\section{Figures}

Figure 3.1. Conceptual Design for Two-Scintillator Sensor Laboratory Test Jig, Enclosed in Light-Tight Box.

Figure 3.2. Electronics Diagram for Laboratory Tests of Two-Scintillator Sensor.

Figure 3.3. Schematic of Laboratory Setup for Preliminary Small-Scintillator Experiments

Figure 3.4. Gamma Spectra Recorded in a Small (10-cm [4-in.] by 10-cm [4-in.] by 1.5-cm [0.6-in.])

Paddle Scintillator

\section{Tables}

Table 2.1. Influence of Medium Surrounding Scintillator on Light Collection Efficiency

Table 2.2. Comparison of 5-cm (2-in.) and 12.7-cm (5-in.) PMT Light-Collection Efficiencies............ 2.5

Table 2.3. Light Collection Efficiencies for Various Light-Guide Configurations ...............................2.6

Table 2.4. Comparison of PMT Light Collection at Scintillator Edge and Corner. 2.7 


\subsection{Introduction}

The U.S. Department of Energy established the Advanced Large-Area Plastic Scintillators (ALPS) project to develop a large-area radiation detector incorporating layered plastic scintillators, optimized lightcollection and signal-processing electronics, and advanced data-analysis algorithms, with the primary technical objective of extracting as much low-energy gamma sensitivity and radiological sourcecharacterization information from plastic scintillators as possible. Pacific Northwest National Laboratory (PNNL) is investigating technology to serve as a model for the "next generation" of large-area plastic scintillant-based detectors, with particular emphasis on enhancing the capabilities of portal monitors. A combination of numerical simulation, laboratory tests, and field deployment will support the design, fabrication, and evaluation of a demonstration sensor.

Project activity commenced in mid-January 2003. Progress to date (30-Apr-03) has focused on the issues of light-collection optimization, conceptual design of a demonstration sensor, and setup of laboratory equipment and test procedures for early stages of benchmarking numerical simulations of scintillator response to a variety of gamma and beta sources. These items are described more fully in the sections that follow. 


\subsection{Light-Collection Optimization}

\subsection{Motivation}

Plastic scintillant offers an attractive gamma-detection medium for large-area sensor applications because it is physically robust and relatively inexpensive per unit area in comparison to $\mathrm{NaI}$ and high-purity germanium (HPGe) crystal detectors. Limited resolution presents the major drawback to plastic scintillators in comparison to the crystal detectors that find widespread use for gamma spectroscopy applications. In part, the resolution of plastic scintillator as a spectroscopic medium arises from an intrinsic limitation in the number of information carriers, i.e. light quanta, created via energy deposition by ionizing radiation interacting in the scintillant. Typical values for the energy required per information carrier are $100 \mathrm{eV}$ per photon for plastic scintillant, $26 \mathrm{eV}$ per photon for $\mathrm{NaI}(\mathrm{Tl})$, and $3 \mathrm{eV}$ per charge carrier in HPGe (Knoll 2000). Since the (statistical) resolution varies as the inverse square root of the number of information carriers, the intrinsic resolution of plastic scintillant should be about a factor of 2 poorer than $\mathrm{NaI}(\mathrm{Tl})$ and about a factor of 6 poorer than HPGe.

The actual energy resolution measured in a given detector will reflect a number of factors beyond the intrinsic statistical resolution considered here, and in the case of the two scintillants considered above, plastic and $\mathrm{NaI}(\mathrm{Tl})$, light-collection efficiency and the conversion of light to electronic pulses for data collection can play a significant role.

To illustrate this, we contrast the light-collection properties of two scintillation detectors, each equipped with a single photomultiplier tube (PMT) with quantum efficiency, i.e. efficiency for converting incident photons into photoelectrons, on the order of $25 \%$. One detector is a standard 7.6-cm (3-in.) length by 7.6$\mathrm{cm}$ (3-in.) diameter right circular cylinder of $\mathrm{NaI}(\mathrm{Tl})$ crystal with a high light-collection efficiency, which (for the sake of argument), we take to be roughly $90 \%$. The second detector is a large-area (on the order of $1-\mathrm{m}^{2}\left[10.8-\mathrm{ft}^{2}\right]$ ) slab of plastic scintillator, with relatively poor light-collection efficiency on the order of $1 \%$. Assuming equal energy deposition of $1 \mathrm{MeV}$ in each detector, the average number of photoelectrons produced in the PMTs viewing the $\mathrm{NaI}(\mathrm{Tl})$ and plastic scintillator sheet will be 8650 and 25 , respectively, yielding resolutions of $1.1 \%$ and $20 \%$ for the two detectors. Thus the "intrinsic" factor of two advantage in resolution enjoyed by the $\mathrm{NaI}(\mathrm{Tl})$ has been amplified in this hypothetical comparison to a factor of close to 20 because of the light-collection shortfall in the large-area plastic scintillator.

This type of consideration argues strongly for investigating light-collection optimization in a large-area plastic scintillator sensor as a promising first step in maximizing sensitivity. The advantages of optimized light-collection efficiency for gamma detection are expected to be threefold: (1) The "gross efficiency" of a sensor, i.e. fraction of incident gammas of a given energy that yield at least one photoelectron in at least one PMT, will increase as the light-collection efficiency increases, for fixed scintillator thickness. This arises because, on average, a smaller energy deposition is required to produce enough photons such that the "survival probability" to at least one PMT is appreciable. (2) Greater flexibility in pulse-processing options, including a requirement of time coincidence between pairs of signals registered from a multiPMT system, accrues with increased light-collection efficiency. This is caused by the increase in probability that at least one photoelectron will be produced in two or more PMTs. The coincidence requirement in turn greatly suppresses background arising from PMT dark current, allowing the effective 
energy-detection threshold for the sensor system to be set at the lowest possible value. (3) Source characterization algorithms employed for existing plastic scintillator-based portal monitors typically employ counts registered by the sensor above a series of several (typically four or less) energy thresholds. The statistical precision or "sharpness" of the sensor counts acquired in a given sampling time will depend upon the scintillator energy resolution. Improving the light-collection efficiency implies an improved scintillator energy resolution, which in turn improves the statistical precision in the energythreshold count patterns processed by the sensor's threat-identification software algorithms. The net effect is that threat identifications can be made at a greater statistical confidence level with a measurement of the same time duration.

\subsection{Light-Collection Modeling: Descriptive}

With these motivating factors in mind, the project commenced with a series of numerical modeling investigations of light-collection efficiency in large-area plastic scintillators. In view of the complexity of the phase space integrations that must be performed to achieve an analytic solution of the general problem of geometric light ray transmission through an optical system, and given the probabilistic elements, e.g. absorption, Rayleigh scattering, and dielectric-dielectric boundary transmission and reflection probabilities, that can influence the fate of a light ray in an optical system, Monte Carlo simulation presents an attractive and readily implemented technique for estimating light-collection properties of a scintillator-based detection system.

Two widely-used codes have been employed in this study, with cross-checks between the output of different codes to establish consistency and reliability of the numerical results. Heaviest emphasis has been placed on the use of the Geant 4 suite of radiation-transport and optical photon-transport codes. Geant $4{ }^{(a)}$ is a CERN C++ based application development framework that permits quite general modeling of radiation transport and optical photon propagation with a flexible package of detector geometry, source specification, and event processing utilities. Use of the development framework requires writing a series of source code files and compiling an application, in contrast to the codes below, that simply read a usersupplied input-deck file. A second code used in the modeling was DETECT 2000, ${ }^{(\mathrm{b})}$ an optical photontransport Monte Carlo based on the earlier DETECT 98 developed by TRIUMF's ${ }^{(\mathfrak{c})}$ PET group; the code has its roots in earlier versions written by Glenn Knoll. This code permits the specification of opticalsystem geometry with several standard shapes (planes, cylinders, etc.) and specification for initial photon source distribution. Finally, a code to calculate transmission through an "adiabatic" light guide of elliptical cross-section with continuously varying major and minor axes - a shape that could not be modeled conveniently with existing codes - was written especially for this project at PNNL. Further details on these calculations are included below.

All sensor configurations modeled can be described generically in the following terms: A parallelepiped (i.e., box-like) slab of plastic scintillant, with user-specified index of refraction and light attenuation length, is coupled to a series of $1,2, \ldots, \mathrm{N}$ PMTs positioned at various points on the perimeter of the scintillator, either with or without light guides between the scintillant and the PMTs. In addition to box scintillator geometries, a version of the Geant 4 simulation handled a modified parallelepiped shape in

(a) wwwinfo.cern.ch/asd/geant4/geant $4 . h t m l$

(b) www.gel.ulaval.ca/detect/

(c) Tri-University Meson Facility, Vancouver, BC. 
which the scintillator corners are missing. The scintillator and light guides (if present) are either directly coupled to air, wrapped in a metallic foil of user-specified reflectivity, or coated with a diffusively reflecting substance. The primary metric for determining scintillator + PMT effectiveness in these studies was taken to be the average light-collection efficiency obtained for uniform distribution of the light source throughout the volume of the scintillator slab. This is not the only possible criterion for quantifying the merits of a scintillator optical readout scheme. For example, uniformity of light response over the area of the scintillator, rather than maximum average response over this area, could be of paramount concern in some applications.

For the majority of the simulations, the PMTs were treated as simple light-collecting boxes, with optical properties determined strictly by the dielectric-dielectric coupling of the PMT material to the scintillator or light-guide material. In reality, the details of PMT light collection as a function of incident light ray position and angle are complex and depend upon the interaction of an incident light ray, not only in the materials constituting the PMT photocathode and glass window, but also on the arrangement and composition of elements deeper within the tube. This complicated functional dependence arises in part because (1) rays incident on the thin photocathode layer can be reflected many times from the interface between the layer and the surrounding materials (glass or vacuum) before an interaction that produces a photoelectron, and (2) incident rays that were transmitted through the photocathode without interaction can be reflected from the interior elements of the tube and strike the photocathode again. These complex details of internal PMT configuration and light response were generally considered to be beyond the scope of the current modeling investigations. However, in a detection scenario described further below, the effect of imposing a simple, angle-dependent acceptance probability on the phase space of rays incident on the PMT surface was evaluated.

\subsection{Light-Collection Modeling: Results}

The first set of modeling results focuses on the light-collection properties of the scintillators, given a certain coupling to the medium that surrounds it - e.g., air, reflecting foil, or a diffusively scattering reflective coating such as aluminum oxide. The various geometrical configurations modeled for these comparisons are described tersely below, with light-collection efficiencies summarized in Table 2.1. Simulation results correspond to runs of 100,000 histories, yielding statistical uncertainties on the resulting light efficiencies, expressed as percentages, of typically less than $0.1 \%$ (absolute).

Configuration A: Geometry consists of a parallelepiped scintillator slab, dimensions $127 \mathrm{~cm}(50 \mathrm{in}$.) by $57 \mathrm{~cm}$ (22.5 in.) by $5 \mathrm{~cm}$ ( 2 in.), with optical properties corresponding to the (quoted) properties of SaintGobain (Bicron) BC-408 scintillator: index of refraction 1.58 and optical photon absorption length $380 \mathrm{~cm}$ (149.6 in.). Light readout is via one 5-cm (2-in.) diameter PMT, directly coupled to the center of one of the short $57-\mathrm{cm}(22.5$-in.) ends. Four scintillator optical finishes were modeled: (1) direct scintillator-to-air coupling (smooth surface polish), (2) "loose" foil wrapping, corresponding to a small air gap between the scintillator surface and the foil wrap to maintain total internal reflection, with specular reflection from the foil (foil reflectivity 85\%), (3) "tight" foil wrapping, corresponding to no air gap between the scintillator surface and the foil (again, specular reflection from the foil with reflectivity $85 \%$ ), and (4) a diffuse-reflection (i.e., Lambertian distributed reflection probability) coating on scintillator, reflectivity 95\%. Direct comparisons between the Geant 4 simulation and the DETECT simulation were possible for three of these cases (air-scintillator coupling, tight foil wrapping, and diffuse 
coating), and in all cases, the two codes agreed to within 0.2 to $0.3 \%$ (absolute) in the light-collection efficiency.

Configuration B: Same as A, but with two 5-cm (2-in.) PMTs positioned at one end of the scintillator.

Configuration C: Same as A, but with two 5-cm (2-in.) PMTs positioned at opposite ends of the scintillator.

Table 2.1. Influence of Medium Surrounding Scintillator on Light Collection Efficiency

\begin{tabular}{|c|c|c|c|c|}
\hline \multirow{2}{*}{ Configuration } & \multicolumn{4}{|c|}{ Light Collection Efficiency (\%) } \\
\cline { 2 - 5 } & $\begin{array}{c}\text { Air-Scintillator } \\
\text { Coupling }\end{array}$ & $\begin{array}{c}\text { Loose Foil } \\
\text { Wrapping }\end{array}$ & $\begin{array}{c}\text { Tight Foil } \\
\text { Wrapping }\end{array}$ & Diffuse Coating \\
\hline A & 2.8 & 3.8 & 1.3 & 1.4 \\
\hline B & 5.4 & 7.0 & 2.7 & 2.1 \\
\hline C & 5.5 & 7.0 & 2.0 & 2.8 \\
\hline
\end{tabular}

Note that in all three configurations, a loose foil wrapping is the most efficient in terms of light collection. For the (relatively small) scintillator edge areas covered by one to two 5-cm (2-in.) diameter PMTs, the collection efficiency scales roughly with the number of PMTs, and in the two-PMT cases, the efficiency is essentially independent of the positioning of the tubes at one end or opposite ends.

The second set of results concerns the variation in light-collection efficiency with the scintillator edge area covered by PMT photocathode(s). The models below contrast the efficiency obtained with 5-cm (2-in.) diameter PMTs and 12.7-cm (5-in.) diameter PMTs, where in all modeling cases, the scintillator is wrapped in "loose foil" of $85 \%$ reflectivity. In addition to the configurations A through C listed above (now generalized to encompass either size PMT), the following additional configurations were modeled:

Configuration D: Same scintillator size and optical properties as in A through C, but with three PMTs at one (short) end of the scintillator.

Configuration E: Same, but with two PMTs at each end, for a total of four tubes.

Configuration F: Same, but with three PMTs at each end, for a total of six tubes.

Table 2.2 summarizes the light-collection efficiencies in each configuration, for 5-cm (2-in.) and 12.7-cm (5-in.) PMTs. The column labeled "Area fraction" refers to the fraction of the total scintillator edge area (two long sides plus two short sides) in contact with the surface of the PMTs. The ratio of the lightcollection efficiency to the area coverage fraction, tabulated in the third column under each tube size, provides a measure of the average potency of increased edge-area coverage in terms of light output. Note that as the area fraction approaches $20 \%$, this ratio drops from a high of about 3.5 (for one 5-cm [2-in.] tube) to about 2 (for six 12.7-cm [5-in.] tubes). This phenomenon can be explained in terms of the detection of reflected rays; a brief discussion is presented in Appendix A. 
Table 2.2. Comparison of 5-cm (2-in.) and 12.7-cm (5-in.) PMT Light-Collection Efficiencies

\begin{tabular}{|c|c|c|c|c|c|c|c||}
\hline \multirow{2}{*}{} & \multirow{2}{*}{$\begin{array}{c}\text { Number } \\
\text { of } \\
\text { PMTs }\end{array}$} & $\begin{array}{c}\text { Area } \\
\text { Fraction } \\
\text { Config. }\end{array}$ & $\begin{array}{c}\text { Light } \\
\text { Collection } \\
\text { Efficiency } \\
\text { (\%) }\end{array}$ & $\begin{array}{c}\text { 5-in. PMT } \\
\text { (Light } \\
\text { Erf(Areac) }\end{array}$ & $\begin{array}{c}\text { Light } \\
\text { Area } \\
\text { Fraction } \\
\text { (\%) }\end{array}$ & $\begin{array}{c}\text { Collection } \\
\text { Efficiency } \\
\text { (\%) }\end{array}$ & $\begin{array}{c}\text { (Light } \\
\text { Eff)/(Area } \\
\text { Frac.) }\end{array}$ \\
\hline A & 1 & 1.1 & 3.8 & 3.5 & 3.3 & 10.9 & 3.3 \\
\hline B & 2 & 2.2 & 7.0 & 3.2 & 6.6 & 19.9 & 3.0 \\
\hline C & 2 & 2.2 & 7.0 & 3.2 & 6.6 & 18.8 & 2.8 \\
\hline D & 3 & 3.2 & 10.4 & 3.2 & 9.9 & 27.4 & 2.8 \\
\hline E & 4 & 4.3 & 13.3 & 3.1 & 13.2 & 30.7 & 2.3 \\
\hline F & 6 & 6.5 & 18.4 & 2.8 & 19.8 & 40.2 & 2.0 \\
\hline \hline
\end{tabular}

A third set of modeling investigations was conducted to evaluate the light-collection properties of light guides and "non-standard" (i.e., non-parallelepiped) scintillator geometries. The modeling of a general light-guide shape presents a geometrical challenge in that light transmission codes typically only permit convenient descriptions of a few geometrical "primitives" (e.g., boxes, cylinder, spheres), whereas light guides in frequent use by the nuclear and particle-physics community often have shapes that cannot be adequately represented by a single primitive, or even a connected series, of these primitives. The "fishtail" design represents one popular type of light guide in which a rectangular surface area (placed in contact with the scintillator edge) transforms continuously into a circular cross-sectional area to match the photocathode shape of a cylindrical PMT. Unfortunately, this design cannot be straightforwardly modeled by either of the codes used for this investigation. To circumvent this difficulty, the following strategy was adopted: The Geant4 application was used to model the response of a detection system in which a "trapezoidal" light guide was positioned between the edge of the scintillator and a (box-shaped, square-faced) PMT. The trapezoidal shape, consisting of one pair of planar faces that are tilted "inward" and one pair tilted "outward" to adapt a rectangular area to a square area, can be easily modeled. In a second modeling step, the light-collection of an ellipsoidal light guide was separately modeled in a Fortran program written especially for this purpose. The ellipsoidal guide approximates the fishtail guide shape by smoothly transitioning from an ellipse at the guide's base (approximately filling the rectangular area at the base of the fishtail guide) to a circular aperture at the apex for connection to a cylindrical PMT. A combination of the Geant4 trapezoidal light-guide results and the independent ellipsoidal guide results then yields an estimate of the light-collection efficiency for a system with fishtail light guides.

The light-guide configurations modeled are listed below. Table 2.3 summarizes the light-collection efficiencies and compares these with the analogous direct-coupling modeling results for the same number of 12.7-cm (5-in.) diameter PMTs. Note that the third column of the table, labeled "Fishtail guide (estimated)," has been obtained from the corresponding trapezoidal guide results in Geant 4 with a correction factor applied as described in the following paragraph.

Configuration G: Same scintillator dimensions, optical properties, and loose-foil wrapping (85\% reflectivity) as in previous configurations. One light-guide was modeled in Geant4, dimensions at base $19 \mathrm{~cm}$ (7.5 in.) by $5 \mathrm{~cm}$ ( 2 in.), dimensions at apex $9.83 \mathrm{~cm}$ (3.87 in.) by $9.83 \mathrm{~cm}$ (3.87 in.) to maintain a $96.8 \mathrm{~cm}^{2}\left(15 \mathrm{in}^{2}\right)$ cross-sectional area. The light guide is wrapped in a loose metallic foil of reflectivity $85 \%$, and positioned at the center of one short (57 cm [22.5-in.]) scintillator end. Photons registered in a 
box-shaped PMT of the same cross-sectional dimensions as the apex of the light guide. (Note: The area of a 12.7 -cm (5-in.) diameter PMT is approximately $126.5 \mathrm{~cm}^{2}\left(19.6 \mathrm{in}^{2}\right)$, so it is assumed that all photons collected in this model would be collected in a $12.7-\mathrm{cm}$ (5-in.) diameter cylindrical PMT.) The correction for the "fishtail" guide was applied by comparing independent Monte Carlo results for transmission through a trapezoidal guide ( $71.3 \%$ transmission for source points uniformly distributed through scintillator volume) and an ellipsoidal guide (94.6\% transmission), yielding an estimated factor of $94.6 / 71.3=1.33$ increase in light-collection efficiency for fishtail guides in comparison with the directlymodeled trapezoidal guide.

Configuration H: Same as G, but with three light guide + PMT detectors positioned along one short edge of the scintillator. Note that the three guides completely cover the 145-cm (22.5-in.) edge area.

Configuration I: Same as H, but with six light guide + PMT detectors, three each along both short edges of the scintillator.

Table 2.3. Light Collection Efficiencies for Various Light-Guide Configurations

\begin{tabular}{|c|c|c|c|c||}
\hline \multirow{2}{*}{ Configuration } & \multirow{2}{*}{$\begin{array}{c}\text { Number } \\
\text { of PMTs }\end{array}$} & $\begin{array}{c}\text { Trapezoidal } \\
\text { Guide }\end{array}$ & $\begin{array}{c}\text { Fishtail Guide } \\
\text { (estimated) }\end{array}$ & $\begin{array}{c}\text { Direct-Coupling of 5 in. PMTs to } \\
\text { Scintillator }\end{array}$ \\
\hline G & 1 & 8.4 & 11.1 & 10.9 (Config. A) \\
\hline H & 3 & 19.8 & 26.3 & 27.4 (Config. B) \\
\hline I & 6 & 26.7 & 35.4 & 40.2 (Config. F) \\
\hline
\end{tabular}

Note that the modeled light-guide efficiencies are consistently poorer than those in the corresponding direct-coupling configuration, despite the increase in scintillator edge-area coverage offered by the guides. This can be understood in terms of transmission losses through the light guides. In configuration $\mathrm{G}$, for example, the contact area of the trapezoidal light-guide base against the scintillator is $98 \mathrm{~cm}^{2}$ $\left(15 \mathrm{in}^{2}\right)$ while the circular surface of a $12.7-\mathrm{cm}(5$-in.) PMT intercepts about $95 \%$ of a smaller rectangular area, $12.7 \mathrm{~cm}$ ( $5 \mathrm{in}$.) by $5 \mathrm{~cm}\left(2 \mathrm{in}\right.$.), or about $61.3 \mathrm{~cm}^{2}\left(9.5 \mathrm{in}^{2}\right)$. However, the roughly $1 / 3$ advantage in surface area enjoyed by the light guide is countered by the transmission losses (on the order of 30 to $40 \%$ ) through the guide, leading to a net decrease in light-collection efficiency relative to the direct-coupling configuration.

Finally, the efficacy of a "corner readout" scintillator geometry was estimated using a modified version of the Geant 4 simulation developed for the parallelepiped scintillator configurations. The modified simulation provides the option of removing the corners from an otherwise box-shaped scintillator, leaving an edge (at $45^{\circ}$ with respect to the adjacent edges) of user-specified length. Box-shaped PMTs can be positioned either along the straight edges or along the corners, with a suitable $45^{\circ}$ rotation of the PMT box to align with the surface of the scintillator. Because the angular distribution of light rays impinging on the PMT photocathode is expected to play a significant role in assessing the relative merits of a cornermounted vs. edge-mounted configuration, the angular acceptance properties of an "idealized" PMT were incorporated in this version of the simulation. A cut on the PMT's angular acceptance was implemented by sampling from a simple gaussian distribution to determine whether a ray incident at an angle $\theta$ with respect to the PMT surface would be accepted as a PMT "hit." The gaussian probability distribution had maximum (unity) transmission probability at normal ray incidence, with a width parameter, sigma, of $45^{\circ}$, 
corresponding to a full-width-half-maximum of about $106^{\circ}$. The simulation kept separate tallies for the number of photons accepted by the PMT with and without the cut. Comparison of angle-of-incidence histograms with and without the cut provided a visual check that the cut was indeed working as expected.

Two configurations were modeled with this corner-less simulation, as described below. The lightcollection efficiencies with and without the angular acceptance cut for both configurations are summarized in Table 2.4. Light-collection efficiencies refer to a 5-cm (2-in.) diameter circular PMT.

Configuration J: Same "standard" scintillator size (127 cm [50 in.] by $57 \mathrm{~cm}$ [22.5 in.] by $5 \mathrm{~cm}$ [2 in.]) and scintillator optical properties as in previous configurations, but with corners of edge length $5 \mathrm{~cm}$ ( 2 in.) removed. The scintillator is wrapped in loose foil of reflectivity $85 \%$. A single box-shaped PMT of dimensions $5 \mathrm{~cm}(2 \mathrm{in}$.) by $5 \mathrm{~cm}$ ( 2 in.) by $5 \mathrm{~cm}(2 \mathrm{in}$.) is positioned at the center of one of the short edges of the scintillator in this configuration. Estimate of light collection in a 5-cm (2-in.) diameter cylindrical PMT is obtained from the simulation results by scaling down the number of simulated PMT hits by the ratio of circular ( $5 \mathrm{~cm}$ [2 in.] diameter) to square $(5 \mathrm{~cm}$ [2 in.] on a side) areas.

Configuration K: Same as previous configuration, but with the box PMT mounted at the corner of the scintillator.

Table 2.4. Comparison of PMT Light Collection at Scintillator Edge and Corner

\begin{tabular}{|c|c|c|c|}
\hline \multirow[b]{2}{*}{ Configuration } & \multirow[b]{2}{*}{ PMT Location } & \multicolumn{2}{|c|}{ Light Collection Efficiency (\%) } \\
\hline & & $\begin{array}{c}\text { Without Angle-of-Incidence } \\
\text { Cut }\end{array}$ & $\begin{array}{c}\text { With Angle-of-Incidence } \\
\text { Cut }\end{array}$ \\
\hline $\mathrm{J}$ & "short" edge & 3.7 & 2.3 \\
\hline $\mathrm{K}$ & corner & 3.5 & 2.4 \\
\hline
\end{tabular}

The net effect of the angle-of-incidence cut, which is intended to more closely model the actual light acceptance of a typical PMT than the simple "light bucket" approach implemented in the simulations described earlier, is to reduce the overall light-collection efficiency by about $30 \%$ to $40 \%$, depending upon the position of the PMT. The efficiency of the corner-mounted PMT is slightly greater $(0.1 \%$ absolute) than the edge-mounted PMT when this effect is taken into account.

\subsection{Light-Collection Modeling: Summary}

The general picture of light collection in large area (on the order of $\sim 1 \mathrm{~m}^{2}\left[\sim 10.8 \mathrm{ft}^{2}\right]$ ) plastic scintillators that emerges from these Monte Carlo simulation studies is one of relatively poor efficiency for "typical" PMT readout configurations, as realized in, e.g., commercially available portal monitors. In the optimal scintillator wrapping case considered here, namely loose foil with an air gap between the scintillator surface and the foil, a single 5-cm (2-in.) cylindrical PMT directly coupled to the edge of a 5-cm (2-in.) thick scintillator will only collect about $4 \%$ of the light released in scintillation events distributed uniformly throughout the scintillator volume. This is true even for the longest optical photon attenuation length (on the order of $4 \mathrm{~m}$ [13 ft]) commonly available from well-established scintillator vendors such as Saint-Gobain/Bicron and Eljen Technologies, Inc. For a rectangular geometry, the light-collection efficiency for a small number (1 or 2) of PMTs shows little dependence on the PMT position around the scintillator edge. Increasing the scintillator edge-area coverage can yield substantially larger light- 
collection efficiencies, reaching a maximum of roughly $40 \%$ for six $12.7-\mathrm{cm}$ (5-in.) diameter directcoupled PMTs (about 20\% scintillator edge-area coverage) in the cases studied here. Light guides appear to be of limited utility in increasing the light-collection efficiency for a thick scintillator because transmission losses through the guides tend to predominate over the increase in scintillator edge-area coverage they offer. Finally, the use of corner-mounted PMT configurations appears to yield a fairly small light-collection advantage over the standard edge-mounted geometry. The study of the effect of limited PMT ray acceptance as a function of incident angle highlights the fact that light-collection efficiencies as quantified in the "light bucket" approach employed in the majority of the simulations reported here (specifically, configurations A through I) may overestimate actual efficiencies by as much as a factor of 1.4 in edge-mounted geometries. However, it should be noted that the relative increase in light-collection efficiency as a function of scintillator edge-area coverage should be independent of this effect to a good approximation, to the extent that a very similar photocathode angular response is common to all PMT models (both $5 \mathrm{~cm}$ [2 in.] and $12.7 \mathrm{~cm}$ [5 in.] diameter, for example), which might realistically be employed for large-area scintillator readout. 


\subsection{ALPS Conceptual Design and Experimental Campaign}

The conceptual design for the ALPS demonstration sensor consists of two sheets of Saint-Gobain/Bicron BC-408 scintillator of dimensions $127 \mathrm{~cm}$ (50 in.) by $57 \mathrm{~cm} \mathrm{(22.5)} \mathrm{in.} \mathrm{by} 5 \mathrm{~cm} \mathrm{(2} \mathrm{in.)} \mathrm{(roughly} 0.725 \mathrm{~m}^{2}$ [7.8 $\left.\mathrm{ft}^{2}\right]$ area per sheet). A set of 12.7-cm (5-in.) diameter Hamamatsu PMTs will be directly coupled to both short (57-cm [22.5-in.]) ends of each scintillator. Sufficient tubes will be acquired to permit outfitting each sheet with six tubes, three at both ends. The modeling calculations described in Section 1.0 above suggest that this arrangement should yield a light-collection efficiency of approximately $40 \%$ averaged over scintillation events distributed uniformly throughout the scintillator volume, ignoring PMT angle-of-incidence losses. The fraction of scintillator edge area covered by photocathode surface will be approximately $20 \%$ in the six tubes per sheet configuration. Note that the current generation of commercially available portal monitors incorporates one or two 5-cm (2-in.) diameter PMTs for scintillator sheets of (typically) $0.5 \mathrm{~m}^{2}\left(5.4 \mathrm{ft}^{2}\right)$ area, corresponding to approximately $4 \%$ (one tube) and 7\% (two tubes) light-collection efficiency with loose-foil wrapping. For comparison to calculations, a set of six "adiabatic" light guides (i.e., approximately constant cross sectional area over the guide length) in a fishtail geometry, also fabricated by Saint-Gobain/Bicron, will be tested on a single scintillator sheet as well. These light guides will cover essentially the entire area of a scintillator sheet's shorter edges, coupling the edges to the circular photocathode areas of the PMTs.

Experimental work on the ALPS project planned for completion in the near term will consist of a campaign to benchmark the light-collection calculations with a variety of scintillator + PMT configurations. An important goal of these experiments will include characterizing scintillator gamma spectral characteristics as a function of light collection. This in turn will require data collection with a variety of gamma button sources in a fairly comprehensive set of the configurations "A" through "I" outlined in Section 1.0, culminating in a full complement of six PMTs per scintillator sheet. Figure 3.1 shows a conceptual design for a laboratory testing jig and light-tight box, with two of the $0.725-\mathrm{m}^{2}$ $\left(7.8-\mathrm{ft}^{2}\right)$ sheets in place and mounted with four PMTs per scintillator. Figure 3.2 presents a schematic view of the signal readout and pulse processing electronics that will be implemented for the laboratory (bench-top) version of the demonstration sensor. Note that in this diagram, "A" and "B" label sets of PMTs are at opposite ends of one scintillator, the "AB" scintillator; this notational scheme is duplicated for the second, "CD" scintillator. Data acquisition for the laboratory phase of the project is based on the CAMAC and NIM nuclear instrumentation standards. Key features of the signal-processing chain include the following:

- pulse-height information from each PMT registered in a charge-integrating analog to digital converter (ADC) (i.e., "QDC”)

- pulse arrival time relative to a selected timing fiducial (e.g., one of the PMTs)

- coincidence logic for determining end-to-end coincidence between a single PMT at one scintillator end and the logical OR of PMTs at the opposite end.

Calculated quantities derivable from data registered in this readout scheme will include the following:

- sum of A and B pulse heights, yielding essentially the "raw" total energy deposition in scintillator $\mathrm{AB}$, and the analogous quantity for scintillator $\mathrm{CD}$ 
- energy deposition corrected for light attenuation in the scintillator, essentially given by the geometric mean of $\mathrm{A}$ and $\mathrm{B}$ pulse heights (with analogous quantities for the $\mathrm{CD}$ scintillator): $E_{\text {corr }}=\left(E_{A} E_{B}\right)^{1 / 2} \exp (\alpha L / 2)$, where $\alpha$ is the optical attenuation length of the scintillant and $L$ is the scintillator length

- position of scintillation event in the plane of the scintillator as derived from pulse-height information: $X_{\text {calc }}=(1 / 2 \alpha) \ln \left(E_{B} / E_{A}\right)$ and $Y_{\text {calc }}=(1 / 2 \alpha) \ln \left[\left(A_{1}+B_{1}\right) /\left(A_{3}+B_{3}\right)\right]$ where $X$ and $Y$ refer to the "long" and "short" axes of the scintillator, respectively

- position of scintillation event in the scintillator plane extracted from (independently calibrated, e.g., via light-emitting diode [LED] probing of the time-delay characteristics of the pulse processing chain) technical data checklist (TDC) information.

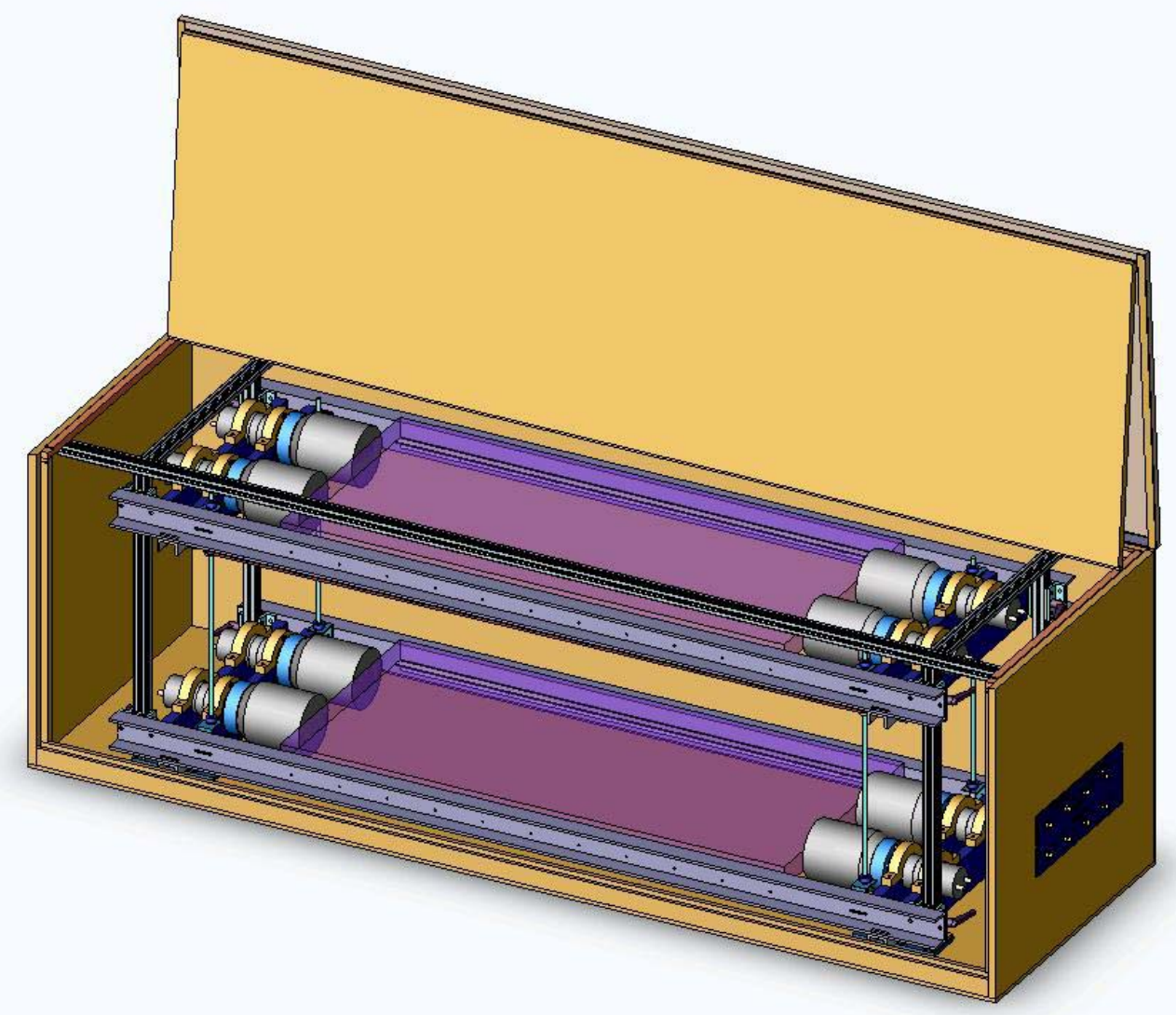

Figure 3.1. Conceptual Design for Two-Scintillator Sensor Laboratory Test Jig, Enclosed in Light-Tight Box 


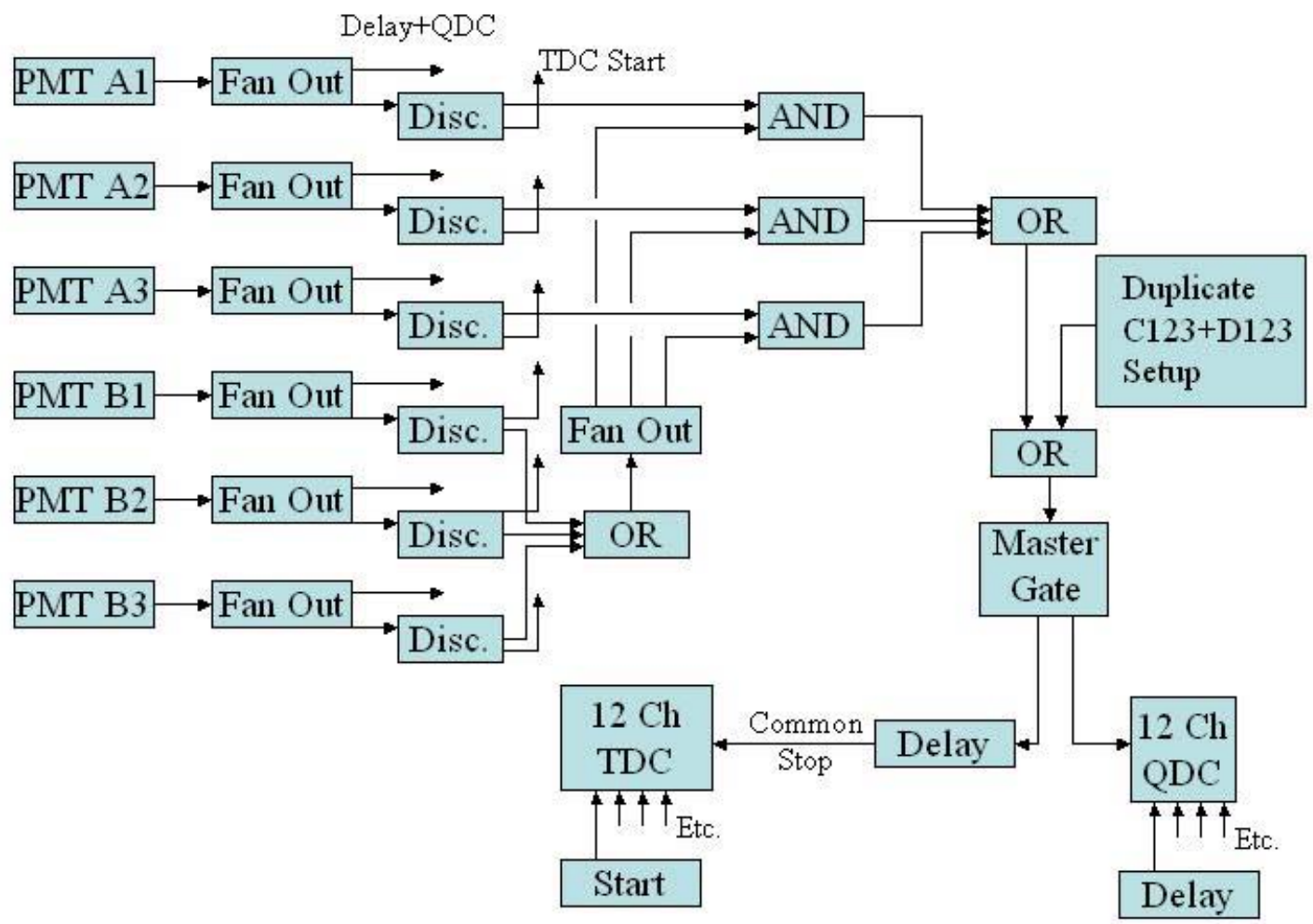

Figure 3.2. Electronics Diagram for Laboratory Tests of Two-Scintillator Sensor.

Experimental work completed to date includes the acquisition of laboratory space, design and/or selection and purchasing of components for the full two-sheet demonstration sensor, and setup and execution of preliminary experiments before (and preparatory for) work with the two-sheet sensor. Laboratory space was established in the limited-area island (LAI) of the PNNL 329 building. This laboratory space features convenient access to existing supplies of NIM and CAMAC electronics and other experimental apparatus. A light-tight box from a previous experiment was secured from storage and used in a series of preliminary experiments, intended primarily to test electronics and data-acquisition methods, but also to serve as a "warm-up" for initial benchmarking of the modeling investigations. The preliminary experiments were conducted using existing equipment, including small representative pieces of plastic scintillator and 5-cm (2-in.) diameter Hamamatsu PMTs. Kmax, a data-analysis and acquisition software tool, was used for data collection in these experiments. The experiments conducted to date include measurements of gamma point sources with a 10-cm (4-in.) by 10-cm (4-in.) by 1.5-cm (0.6-in.) plastic scintillator coupled to a single 5-cm (2-in.) diameter PMT. Figure 3.3 presents a schematic view of the experimental arrangement for these early tests, and Figure 3.4 shows background-subtracted spectra obtained with two of the gamma sources. Analysis of these data and comparison to simulation are in progress at the time this report is being written.

Planned follow-up experiments include end-to-end coincident measurements with a $30-\mathrm{cm}$ (11.8-in.) by 30-cm (11.8-in.) by 6-cm (2.4-in.) scintillator directly coupled to multiple 5-cm (2-in.) diameter PMTs, 
before measurements with the full-sized $\left(0.725 \mathrm{~m}^{2}\left[7.8 \mathrm{ft}^{2}\right]\right)$ scintillator sheets, which are now in hand at PNNL.

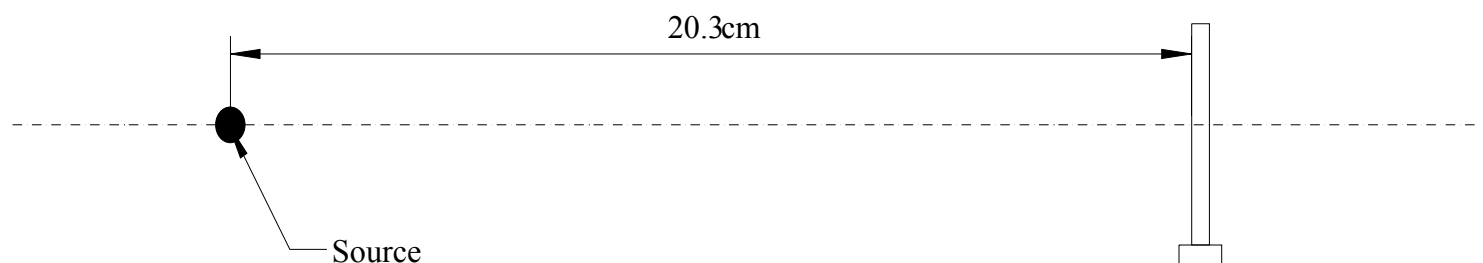

Hamamatsu H1 161-50 PMT

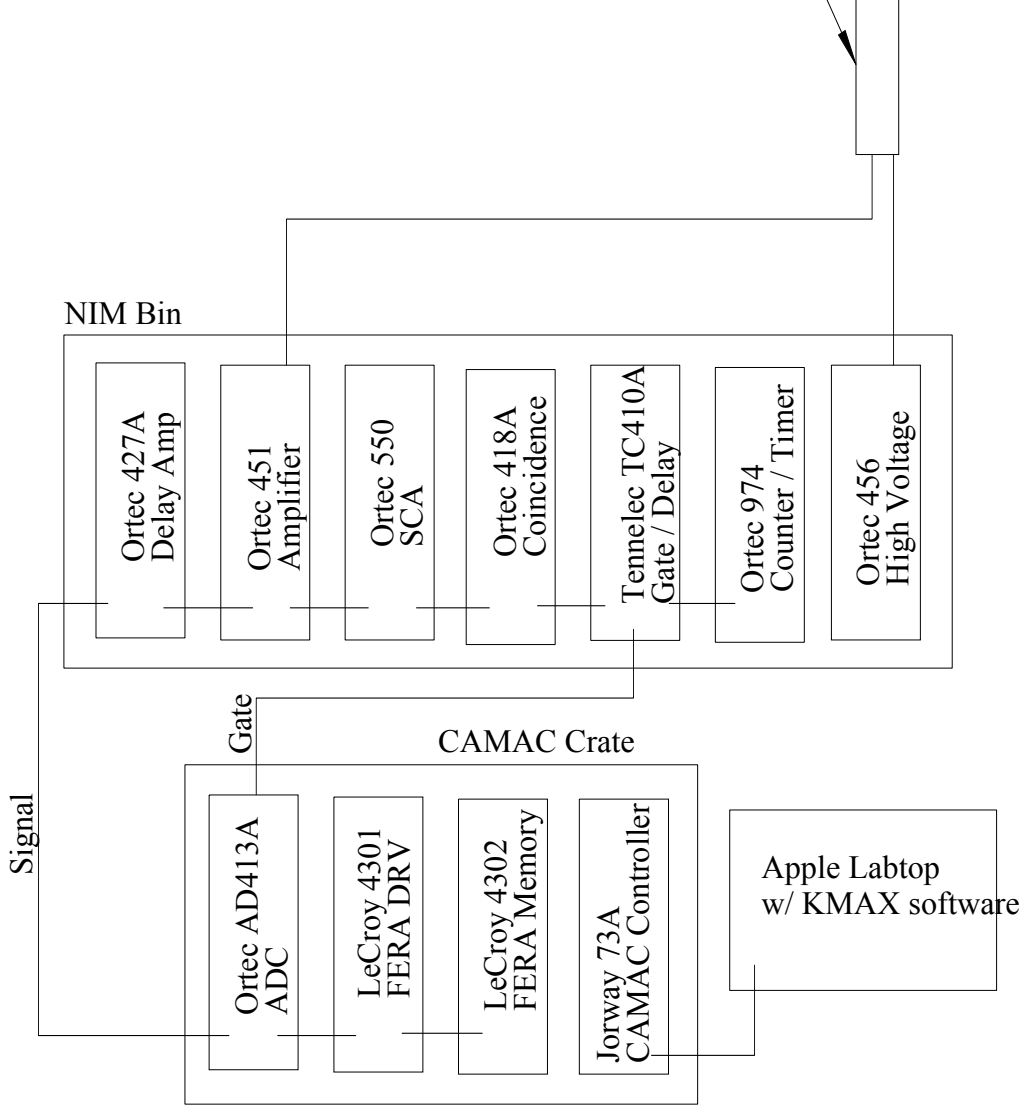

Figure 3.3. Schematic of Laboratory Setup for Preliminary Small-Scintillator Experiments 


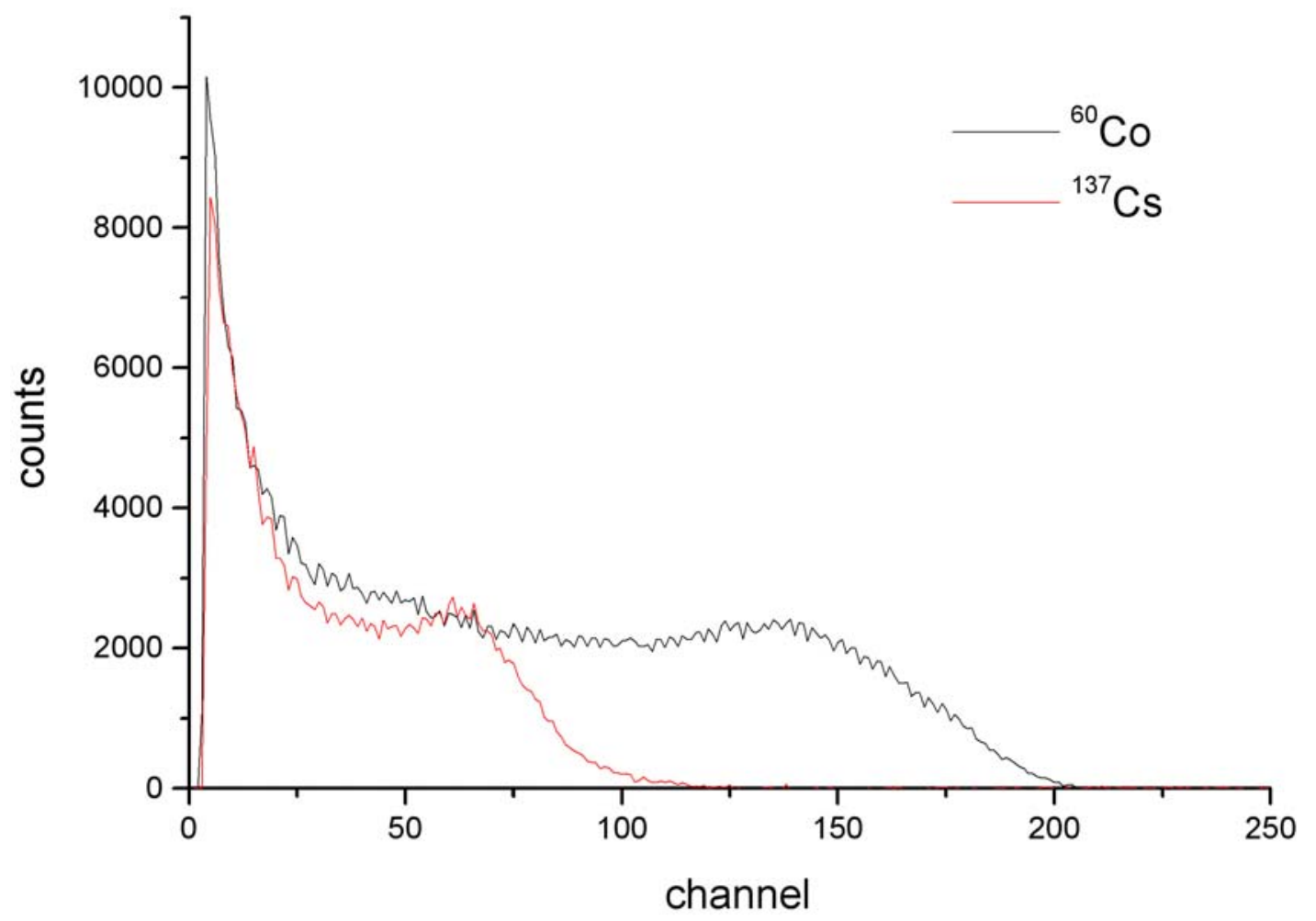

Figure 3.4. Gamma Spectra Recorded in a Small (10-cm [4-in.]

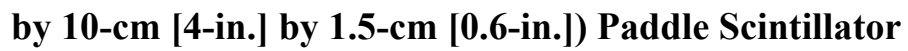




\subsection{References}

Knoll G. 2000. Radiation Detection and Measurement. 3rd edition, Wiley, NY. 


\subsection{Appendix: Light-Collection Efficiency Scaling Effect}

This appendix presents a semi-quantitative explanation for the scaling behavior (law of diminishing returns) of the light-collection efficiency with scintillator edge-area coverage observed in the modeling studies described in Section 2.0. It is reasonable to expect that, in the limit of short optical absorption length, photons can travel only a small distance before either being absorbed in the scintillator medium or impinging on the photocathode of a PMT. In this case, only those photons produced close to a PMT can contribute to the detected light fraction, and thus the collection efficiency will scale linearly with the edge-area coverage. However, in the case of a relatively long absorption length (i.e., comparable to or greater than the maximum linear dimension characterizing the scintillator), where an appreciable fraction of rays reflected from one edge of the scintillator can travel the length of the plastic sheet and impinge on a detection surface at the opposite edge, the fraction of rays detected will, in general, not scale linearly with the fractional area coverage. Consider first the case of a single PMT directly coupled to the center of one "short" end of a scintillator with long (order meters) attenuation length. The PMT views not only those rays that are emitted directly toward its photocathode, but also those rays that reflect off the edges of the scintillator, perhaps several times, before arriving at its surface. In effect, the light-collection effectiveness of this single PMT is amplified by the reflection effect so that a larger fraction of light rays is collected than would otherwise be expected from solid-angle considerations alone.

Next consider the effect of adding additional PMTs to the scintillator edge, thus increasing the fractional edge-area coverage. To quantify the effect of the increase, divide all light rays produced in the scintillator into three classes. The first class consists of those rays which, in the absence of a PMT surface, would reflect from one of the short scintillator ends and reach the opposite short end. The second class consists of rays that are either absorbed in the scintillant or in the wrapping (or are transmitted to the surrounding medium in the case of direct scintillator-to-air coupling) after only a single reflection from one of the short ends. The third class consists of rays that are absorbed or transmitted out of the scintillator without reaching either of the short ends. Note that the number of detected rays in the first class will be very roughly constant as PMTs are added to the two short edges because a PMT at either end will detect the photon. That is, whether the photon strikes a PMT at one end or the other, it will be detected by one of them, and the increase in area coverage afforded by an additional PMT does not substantially increase the number of photons detected. This approximation becomes increasingly exact for this first class of rays as the fraction of area coverage for the two short ends approaches $100 \%$. Detected photons in the second class will scale roughly as the total edge area covered, per the argument advanced in the first paragraph of this appendix. And, of course, rays in the third class will not contribute to the number of detected photons. Thus, roughly speaking, the total fraction of detected photons varies as $\mathrm{C}_{1}+\mathrm{C}_{2} \mathrm{~A}$, where $\mathrm{C}_{1}$ and $\mathrm{C}_{2}$ are constants corresponding to the first and second classes described above, and $\mathrm{A}$ is the fractional edge area covered by the PMTs. Then dividing this fraction by the edge-area coverage, A, yields (light collection fraction $) /($ area coverage fraction $)=\mathrm{C}_{1} / \mathrm{A}+\mathrm{C}_{2}$, i.e., the ratio of the light-collection fraction to the fractional edge-area coverage decreases with increasing area coverage, the same trend observed in the modeling. (Clearly this approximate scaling law breaks down for small $\mathrm{A}$, since the expression $\mathrm{C}_{1} / \mathrm{A}$ diverges as A goes to zero.) 